\title{
Superior Effect of the Combination of Carbon-lon Beam Irradiation and 5-Fluorouracil on Colorectal Cancer Stem Cells in vitro and in vivo
}

This article was published in the following Dove Press journal: OncoTargets and Therapy

\author{
Woong Sub Koom ${ }^{1,2}$ \\ Sei Sai iD ${ }^{3}$ \\ Masao Suzuki ${ }^{3}$ \\ Akira Fujimori ${ }^{3}$ \\ Shigeru Yamada ${ }^{2}$ \\ Hirohiko Tsujii ${ }^{2}$ \\ 'Department of Radiation Oncology, \\ Yonsei Cancer Center, Yonsei University, \\ College of Medicine, Seoul, South Korea; \\ ${ }^{2}$ QST Hospital, National Institutes for \\ Quantum and Radiological Science and \\ Technology (QST), Chiba, Japan; \\ ${ }^{3}$ Department of Basic Medical Sciences \\ for Radiation Damages, National Institute \\ of Radiological Sciences (NIRS), National \\ Institutes for Quantum and Radiological \\ Science and Technology (QST), Chiba, \\ Japan
}

Background: The aim of this study was to investigate whether carbon-ion beam irradiation in combination with 5-fluorouracil (5-FU) is superior to carbon-ion beam irradiation alone in targeting colorectal cancer stem-like cells (CSCs).

Materials and Methods: Human colorectal cancer (CRC) cells, HCT116 and HT29, were treated with carbon-ion beam irradiation alone or in combination with 5-FU. Cell viability assay, colony and spheroid formation assay, apoptotic assay, and quantitative real-time PCR analysis of apoptosis- and autophagy-related gene expression were performed.

Results: Carbon-ion beam irradiation dose-dependently decreased CRC cell viability and showed significantly enhanced cell killing effect when combined with 5-FU. Carbon-ion beam irradiation in combination with 5-FU significantly increased the percentage of apoptotic cells. The expression of some apoptotic and autophagy-related genes such as Bax, Bcl2, Beclin1 and $A T G 7$ was significantly induced by carbon-ion beam irradiation alone and was further enhanced when the beam was combined with 5-FU. The spheroid forming capacity of CD133+ cell subpopulations was significantly inhibited by carbon-ion beam in combination with 5-FU. Histopathologically, the combination of carbon-ion beam irradiation and 5-FU destroyed more xenograft tumor cells, and resulted in increased necrosis, cavitation, and fibrosis, compared to carbon-ion beam irradiation alone.

Conclusion: In conclusion, carbon-ion beam treatment combined with 5-FU has the potential to kill CRC cells including CSCs by inducing increased apoptosis and autophagy.

Keywords: colorectal cancer stem cell, heavy ion radiation, 5-FU

\section{Introduction}

Colorectal cancer (CRC) is the most common gastrointestinal cancer and the second leading cause of cancer-related death in Korea and Japan. ${ }^{1,2}$ Surgical resection is the primary treatment for CRC. However, patients have a higher recurrence rate if the primary tumor has penetrated the serosa or if local lymph node metastases are present at the time of surgery. ${ }^{3-6}$ In fact, a high percentage of locally recurrent rectal carcinoma show bone or vessel infiltration in the pelvis, which make surgical resection difficult.

Locally recurrent CRCs are generally radioresistant as they contain large number of hypoxic cells, and it is difficult to elevate the radiation dose due to the proximity to nearby radiosensitive organs, including the colon, bladder, and small intestine. ${ }^{7,8}$ Therefore, an effective combination therapy to eradicate radioresistant tumor cells is urgently needed. ${ }^{9,10}$ Combination of radiation therapy and 5-fluorouracil (5-FU) treatment has been shown to improve the outcome in advanced CRC. ${ }^{11-13}$ Recently, the
Correspondence: Sei Sai

Department of Basic Medical Sciences for Radiation Damages, National Institute of Radiological Sciences (NIRS), National Institutes for Quantum and Radiological Science and Technology (QST), 4-9Anagawa Inage-Ku, Chiba 263-8555, Japan Tel +8I 43-206-3231

Fax +8I 43-206-4I49

Email sai.sei@qst.go.jp 
prognosis for metastatic or unresectable CRC has improved owing to the approval of 5-FU for advanced CRC. ${ }^{14,15}$ Meanwhile, a small population of colorectal cancer stem cells (CSCs) was identified on the basis of the cell membrane markers $\mathrm{CD} 133+$, CD44 +, ESA + cells. ${ }^{16,17}$ CSCs are closely correlated with tumor recurrence, metastasis and chemoradioresistance. $^{18-20}$ Therefore, developing more effective therapies to eliminate CSC is crucial. ${ }^{21-26}$

Based on high local control rate and increased induction of unrepairable clustered DNA damage, we successfully treated more than 13,000 patients with various tumor types, including more than 600 patients with postoperative recurrent $\mathrm{CRC}$ using carbon-ion radiotherapy (CIRT) over the past 26 years and have achieved encouraging outcomes. $^{27-29}$ However, limiting dose escalation in cases where the tumor is very close to important organs or with invisible micrometastases is one of the most important challenges with CIRT. Therefore, carbon-ion beam irradiation in combination with the DNA damaging agent 5-FU not only has the potential to destroy radioresistant $\mathrm{CSCs}$ but can also reduce the radiation dose required, and still destroy $\mathrm{CRC}$.

Recently, it has been reported that carbon-ion beam irradiation alone can effectively kill colorectal and pancreatic CSCs, and when combined with DNA damaging agents such as gemcitabine,cisplatin, tyrosine kinase inhibitor such as lapatinib, and bisphosphonate such as zoledronic acid are more potent in killing various cancer cells including CSCs compared to carbon-ion beam irradiation alone. ${ }^{30-36}$ In the current study, we investigated the effect of carbon-ion beam irradiation in combination with the DNA damaging agent 5-FU, on CRC viability, colony and spheroid formation ability of CSCs, and changes in the expression of cell deathrelated genes. To the best of our knowledge, this is the first study to demonstrate that carbon-ion beam irradiation in combination with 5-FU is more effective in targeting colorectal CSCs compared to carbon-ion beam irradiation alone.

\section{Materials and Methods}

\section{Cell Lines}

Colon adenocarcinoma cell lines HCT116 and HT29 were originally obtained from the American Type Culture Collection (Manassas, VA, USA). Cells were cultured in Dulbecco's Modified Eagle Medium (Invitrogen, Grand Island, NY) supplemented with $10 \%$ fetal bovine serum (FBS, Beit-Haemek, Kibbutz Beit Haemek, Israel) at $37^{\circ} \mathrm{C}$ with $5 \% \mathrm{CO}_{2}$. The medium was changed twice a week.

\section{Irradiation}

High linear energy transfer (LET) carbon-ion beams produced by the heavy ion medical accelerator in Chiba (HIMAC) were used to irradiate cells or mice. Detailed descriptions about the characteristics of the carbon-ion beams, the biological irradiation procedure, and dosimetry have been described previously. $^{27}$ In brief, a $290 \mathrm{MeV} / \mathrm{u}$ carbon-ion beam with a $6 \mathrm{~cm}$ spread-out Bragg peak (SOBP) at the center $(50 \mathrm{keV} /$ $\mu \mathrm{m})$ was used. As a reference, the cells or mice were also irradiated with conventional $200 \mathrm{kV}_{\mathrm{p}}$ X-ray (TITAN-320, GE, Lewistown, PA, USA).

\section{Cell Viability Assay}

CellTiter-Glo Luminescent Cell Viability Assay (Promega, Madison, WI, USA) or WST-1 Cell Proliferation Assay (Roche, Mannheim, Germany) was used for cell viability analysis. The CellTiter-Glo ${ }^{\circledR}$ Luminescent Cell Viability Assay is a standard method for determining the number of viable cells in culture based on the quantification of ATP present, which is indicative of metabolically active cells. The CellTiter-Glo ${ }^{\circledR}$ Assay is ideal for cell proliferation and cytotoxicity assays performed by automated, highthroughput screening in a multi-well plate format. In the homogeneous assay procedure, a single reagent (CellTiter$\mathrm{Glo}^{\circledR}$ Reagent) is added directly to cells grown in serumsupplemented medium. WST-1 Cell Proliferation Assay is a colorimetric analysis of formazan formation in living cells based on the cleavage of the tetrazolium salt WST-1 by mitochondrial dehydrogenase.

\section{CSC Assays}

Clonogenic and spheroid formation assays were performed with the previously established methods ${ }^{30}$ that using cells sorted from HCT116 cells. The sorted CD133+ and CD44 $+/ \mathrm{ESA}+$ cell subpopulations were resuspended at a cell density of 500 or 2000 cells $/ \mathrm{mL}$ and plated in triplicate in $6 \mathrm{~cm}$ dishes or 96-well spheroid formation plates (Sumilon, Sumitomo Bakelite Co., Ltd, Tokyo, Japan). After allowing the cells to grow for 1-2 weeks, the percentage of colonies or spheres per well was calculated and expressed as the percentage of wells containing colonies or spheres.

\section{Analysis of Apoptosis Using Flow Cytometry}

Apoptosis was analyzed using flow cytometry with the Annexin V-FITC Apoptosis Detection Kits (R\&D Systems, Minneapolis, MN USA) according to the 
manufacturer's protocol. Briefly, irradiated cells were harvested after $96 \mathrm{~h}$ by trypsinization, washed with PBS, and apoptotic and necrotic cells were analyzed following the addition of $100 \mu \mathrm{L}$ binding buffer and $1 \mu \mathrm{L}$ fluorescently labeled Annexin V-FITC in each sample. The samples were mixed gently and incubated at room temperature in the dark for $15 \mathrm{~min}$. Immediately prior to the analysis by flow cytometry (BD FACSCalibur Flow Cytometry System), $1 \mu \mathrm{L}$ of propidium iodide (PI, $1 \mathrm{mg} / \mathrm{mL}$; Cedarlane Laboratories, Hornby, Ontario, Canada) was added to each sample. A minimum of 10,000 cells were analyzed in the gated area.

\section{Real-Time RT-PCR Analysis of Expression of Various Genes Related to Apoptosis and Autophagy}

RNA was extracted using the Qiagen RNeasy kit following on-column DNAse treatment to remove genomic DNA. cDNA was reverse transcribed using the $\mathrm{RT}^{2}$ First Strand Kit (SABiosciences, Frederick, Maryland, USA). Apoptotic- and autophagy-related gene expression was analyzed using real-time RT-PCR on a LightCycler ${ }^{\circledR} 96$ system (Roche, Basel, Switzerland). Data analysis was performed by applying the $\Delta \Delta \mathrm{Ct}$ method using the RTPCR software package $(n=3) \cdot{ }^{31,32}$ The primer sequences used in the PCR are shown in Table 1. GAPDH was used as a housekeeping gene.

\section{Histopathological Analysis of Xenograft Tumor Following Carbon-lon Beam} Irradiation Alone or in Combination with

\section{5-FU}

NOD/SCID mice (male, 6-8 weeks old, Charles River Laboratories, Japan) were housed under conditions defined in the National Institutes for Quantum and Radiological Science and Technology (QST)-National Institute of Radiological Sciences (NIRS) animal facility. HCT116 cells were injected in the right legs of the NOD-SCID mice, and the mice were treated with carbon-ion beam alone or in combination with 5-FU once the xenograft tumors reached approximately $10 \mathrm{~mm}$ in diameter. Following carbon-ion beam irradiation, 5-FU was administered through intraperitoneal injection (I.P) twice a week for 2 weeks. Mice were sacrificed at designated time intervals (4-10 weeks) after treatment with carbon-ion beam irradiation alone or in
Table I The Primer Sequences for Real-Time PCR

\begin{tabular}{|c|c|}
\hline Gene & Primer Sequence \\
\hline \multicolumn{2}{|l|}{ GAPDH } \\
\hline Forward & 5'- TGAACGGGAAGCTCACTGG-3' \\
\hline Reverse & 5'- TCCACCACCCTGTTGCTGTA-3' \\
\hline \multicolumn{2}{|l|}{ Bax } \\
\hline Forward & 5'- CAAACTGGTGCTCAAGGCC-3' \\
\hline Reverse & 5'- GCACTCCCGCCACAAAGAT-3' \\
\hline \multicolumn{2}{|l|}{ BCl-2 } \\
\hline Forward & 5'- ATGTGTGTGGAGAGCGTCAACC-3' \\
\hline Reverse & 5'- TGAGCAGAGTCTTCAGAGACAGCC-3' \\
\hline \multicolumn{2}{|l|}{ LC3-II } \\
\hline Forward & 5'- GATGTCCGACTTATTCGAGAGC-3' \\
\hline Reverse & 5'- TTGAGCTGTAAGCGCCTTCTA-3' \\
\hline \multicolumn{2}{|l|}{ Beclin-I } \\
\hline Forward & 5'- AGCTGCCGTTATACTGTTCTG-3' \\
\hline Reverse & 5'- ACTGCCTCCTGTGTCTTCAATCTT-3' \\
\hline \multicolumn{2}{|l|}{ ATG-7 } \\
\hline Forward & 5'- GCTG CTACTTC TGCAATGATGT -3' \\
\hline Reverse & 5'- GCAAGCTCACTAGG CTGCAGAACC-3' \\
\hline
\end{tabular}

combination with 5-FU. Tissues were fixed in formaldehyde and histopathologically analyzed using hematoxylin and eosin (HE) staining. All the animal experiments were conducted in or accordance with the QST-NIRS Institutional Animal Welfare Guidelines and were approved by the Institutional Animal Care and Use Committee of QST (Approval number:19-2006).

\section{Statistical Analysis}

StatView software (SAS Institute, Inc., Cary, NC, USA) was used to perform one-way analysis of variance and Bonferroni's multiple comparisons test for differences in the mean between groups. $p<0.05$ was considered significant in all comparisons.

\section{Results}

\section{Cell Viability Following Treatment with Different Concentration of 5-FU}

To examine the cytotoxicity of 5-FU in HCT116 and HT29 cells, the cells were treated with different concentrations of 5-FU for different time points, then the cell viability was determined using WST-1 assay and CellTiter-Glo $^{\circledR}$ luminescent cell viability assay. We found that there was no significant dose-dependent 
A

HCT116

HT29

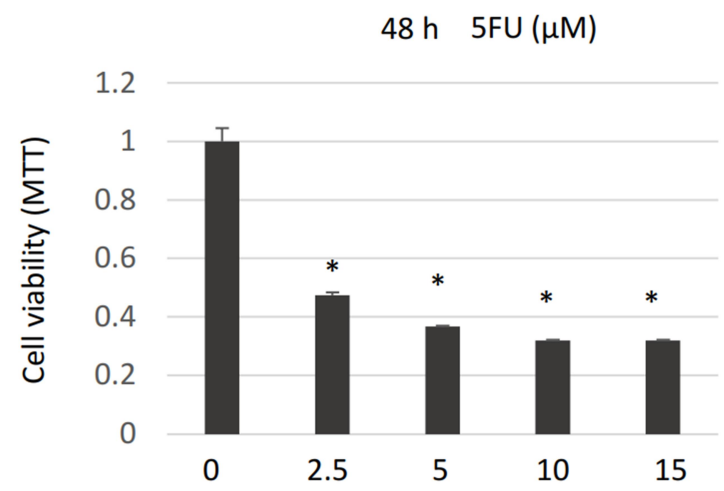

B

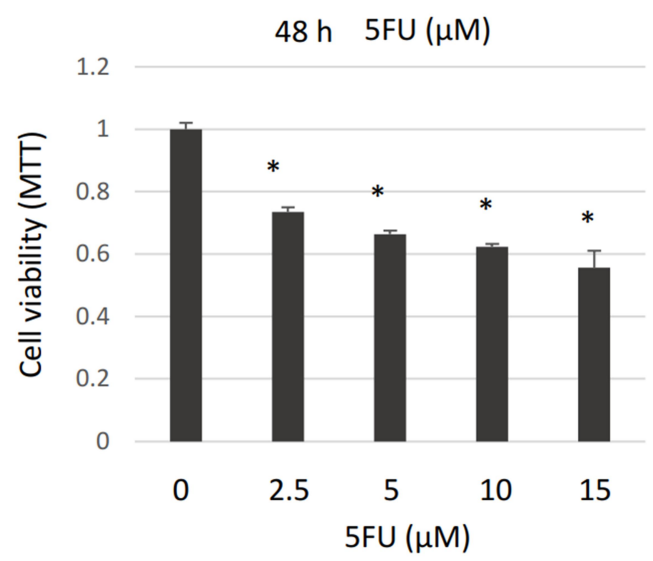

HCT116

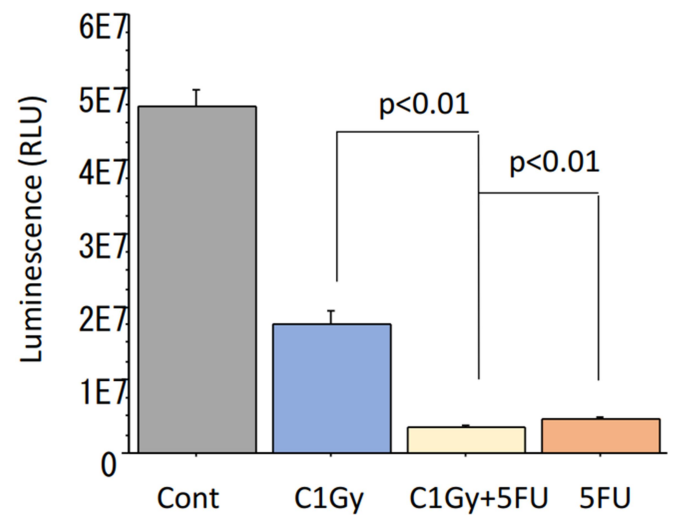

HT29

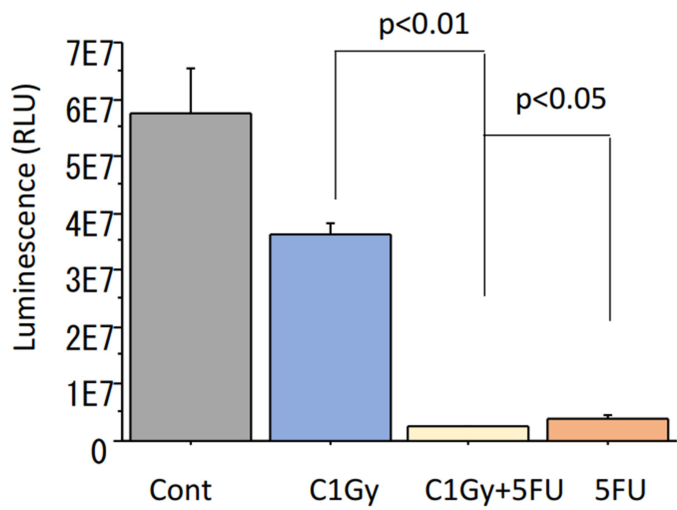

Figure I (A) Viability of HCTII6 and HT29 cells treated with different concentration of 5-FU $(2.5,5,10$, and I5 I5 $\mu$ M)) for 48 h was determined by WST-I assay. (B) Viability of HCTII 6 and HT29 cells treated with carbon-ion beam irradiation alone or in combination with 5 -FU for 96 h was determined by CellTiter-Glo ${ }^{\circledR}$ Luminescent Cell Viability Assay. *, $\mathrm{p}<0.01$ compared to control.

effect at $24 \mathrm{~h}$, but cell viability was significantly decreased 48 and $72 \mathrm{~h}$ after treatment with 5-FU (Figure 1A).

\section{Cell Viability Following Treatment with Carbon-Ion Beam Alone or in Combination with 5-FU}

To determine radiosensitizing effects of 5-FU in HCT116 and HT29 cells, the cells were irradiated with carbon-ion beam alone or in combination with 5-FU, and cell viability was analyzed using CellTiter-Glo ${ }^{\circledR}$ luminescent cell viability assay. As shown in Figure 1B, cell viability of both HCT116 and HT29 cells was reduced following treatment with carbon-ion beam and
5-FU treatment alone, and further reduced following combination treatment.

\section{Apoptosis Induction Following Carbon-Ion Beam Irradiation Alone or in Combination with 5-FU}

To examine apoptosis induction in HCT116 and HT29 cells following carbon-ion beam irradiation alone or in combination with 5-FU. FACS analysis was performed using AnnexinV-FITC/PI detection kit. As shown in Figure 2, carbon-ion beam irradiation significantly enhanced early and total apoptosis in a dose-dependent manner, which was markedly increased when the irradiation was combined with 5-FU in HCT116 (Figure 2A) and HT29 cells (Figure 2B). 
A
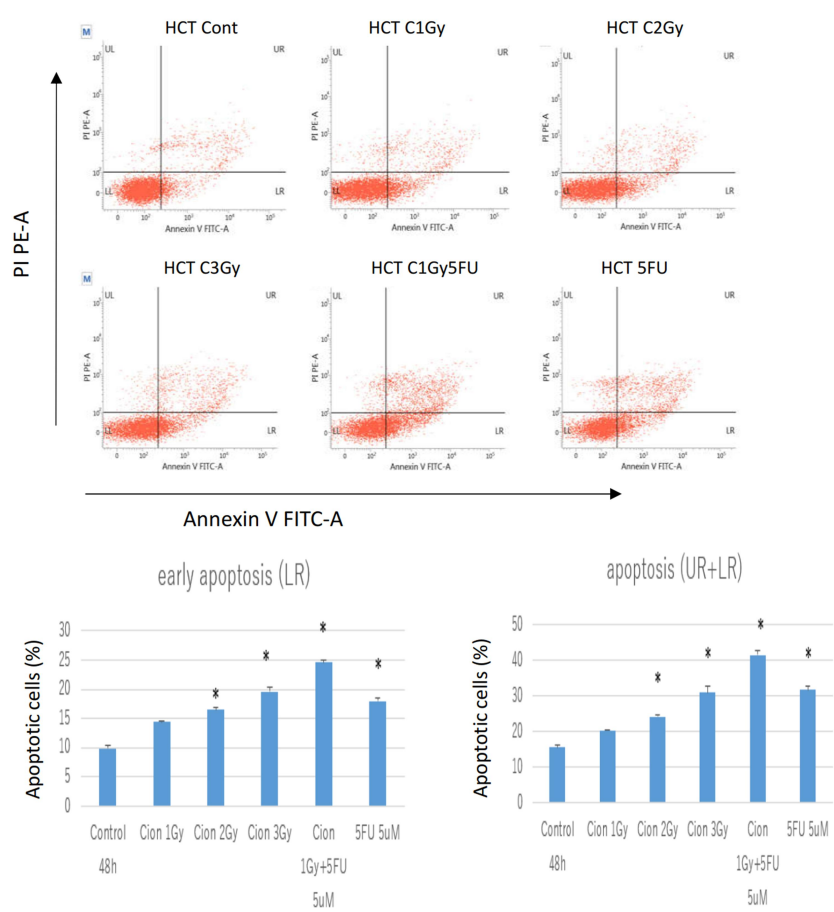

B
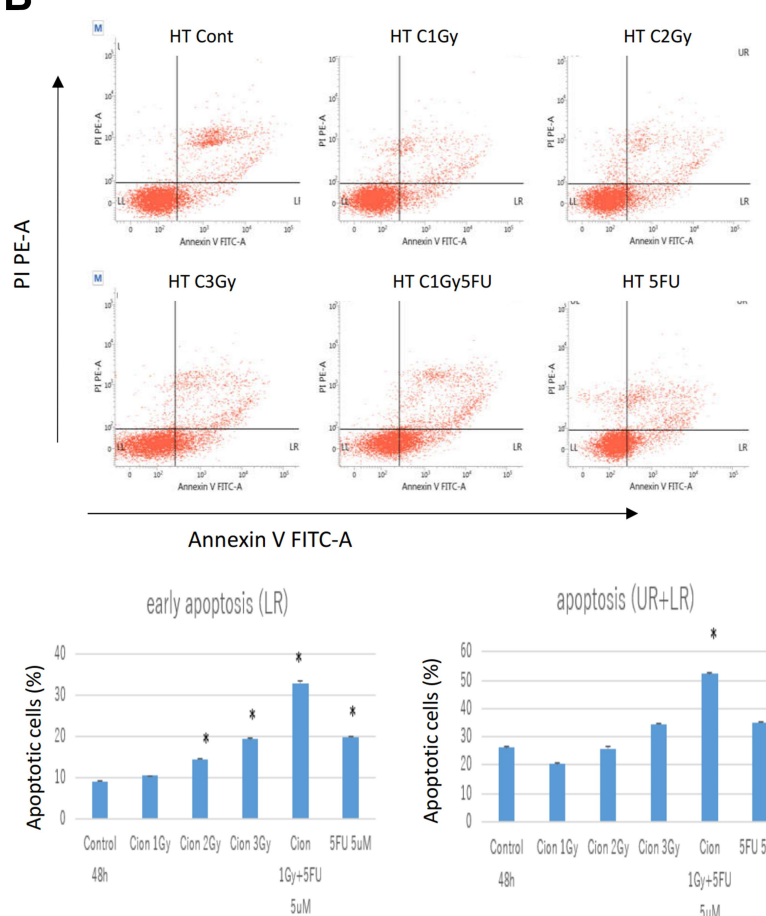

Figure 2 Induction of apoptosis in HCTII6 (A) and HT29 (B) cells treated with carbon-ion beam irradiation alone or in combination with 5 -FU for 96 h. Apoptosis was detected by Annexin-PI kit using FACS analysis. *, $\mathrm{p}<0.01$ compared to control.

\section{CSC Characteristics of CDI33+ and CD44+/ESA+ Cells Sorted from HCTII6}

The CSC properties of CD133+ and CD44+/ESA+ cells were confirmed using the same method described previously. ${ }^{27}$ Representative immunofluorescence stained CSCs (CD133+ and CD44+/ESA+ cells) were shown in Figure 3A. Following plating of equal number of cells (500 cells), CD133+ and CD44+/ESA+ cells sorted from HCT116 formed significantly higher number of colonies (Figure 3B) and large-sized spheres (Figure 3C) compared to that formed from CD133- and CD44-/ESA- cells $(p<0.01)$. The in vivo tumorigenicity assay showed that only CD133+ and CD44 +/ESA+ cells formed tumors, whereas CD133- and CD44-/ ESA- cells did not form tumors when subcutaneously injected to NOD-SCID mice (Figure 3D).

\section{Spheroid Forming Ability of CDI33+ and CD44+/ESA+ Cells Derived from HCTII 6 and HT29 Cells Treated with Carbon-Ion Beam, X-Ray Alone or in Combination with 5-FU}

The number of colonies was markedly reduced when carbon-ion beam irradiation was combined with 5-FU compared to when cells were treated with carbon-ion beam alone or X-ray combined with 5-FU (Figure 3E). We also examined the ability of CSC (CD133+, CD44 +/ESA+) and non-CSCs (CD133-, CD44-/ESA-) to form spheroids following carbon-ion beam irradiation alone or in combination with 5-FU. As shown in Figure 3E, the size of spheroids formed in CSCs sorted from HCT116 and HT29 cells was significantly reduced following treatment with carbon-ion beam irradiation alone and was significantly further reduced when carbon-ion beam was combined with 5-FU. In contrast, a small size of sphere formed in non-CSCs has shrunk significantly following treatment with carbon-ion beam irradiation alone and was enormously reduced following combination treatment with carbon-ion irradiation and 5-FU.

\section{Changes in the Expression of Multiple Genes Following Carbon-Ion Beam, $X$-Ray Irradiation Alone or in Combination with 5-FU}

To examine and compare the changes in the expression of multiple genes, the unsorted HCT116 cells were irradiated with carbon-ion beam or X-ray alone or in combination 


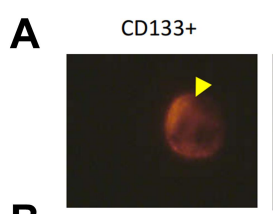

B

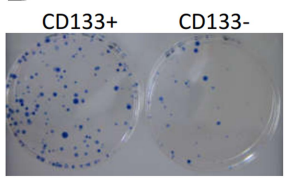

CD44+/ESA+ CD44-

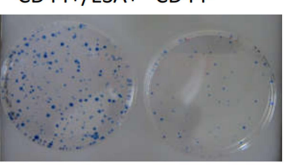

c
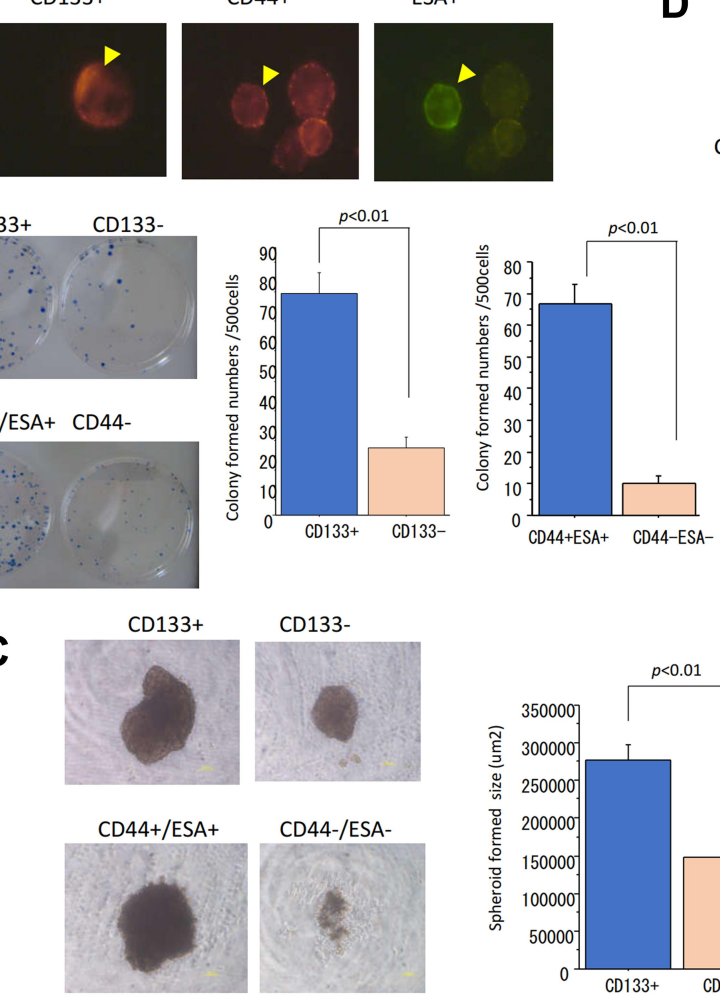

D133-

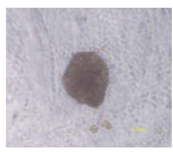

CD44-/ESA-

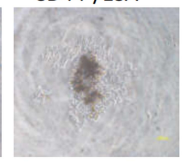

E
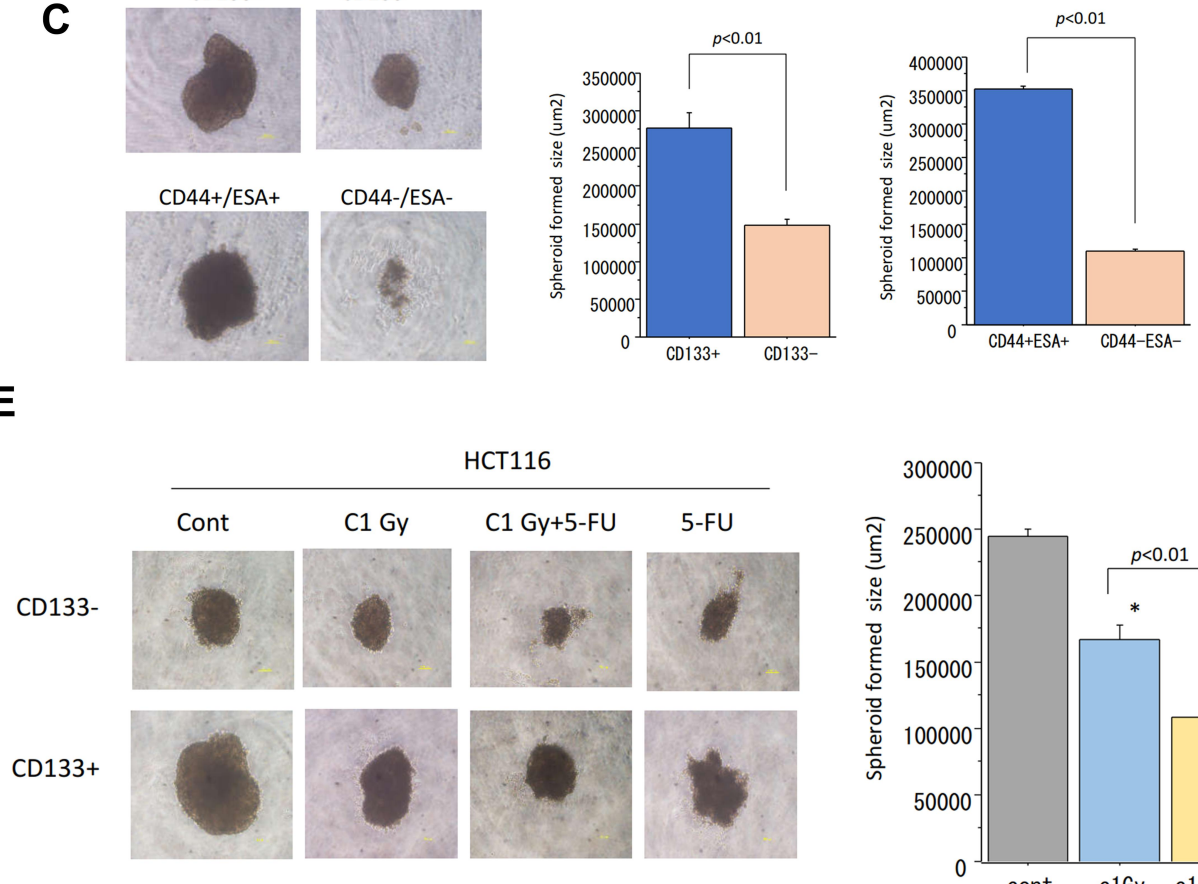
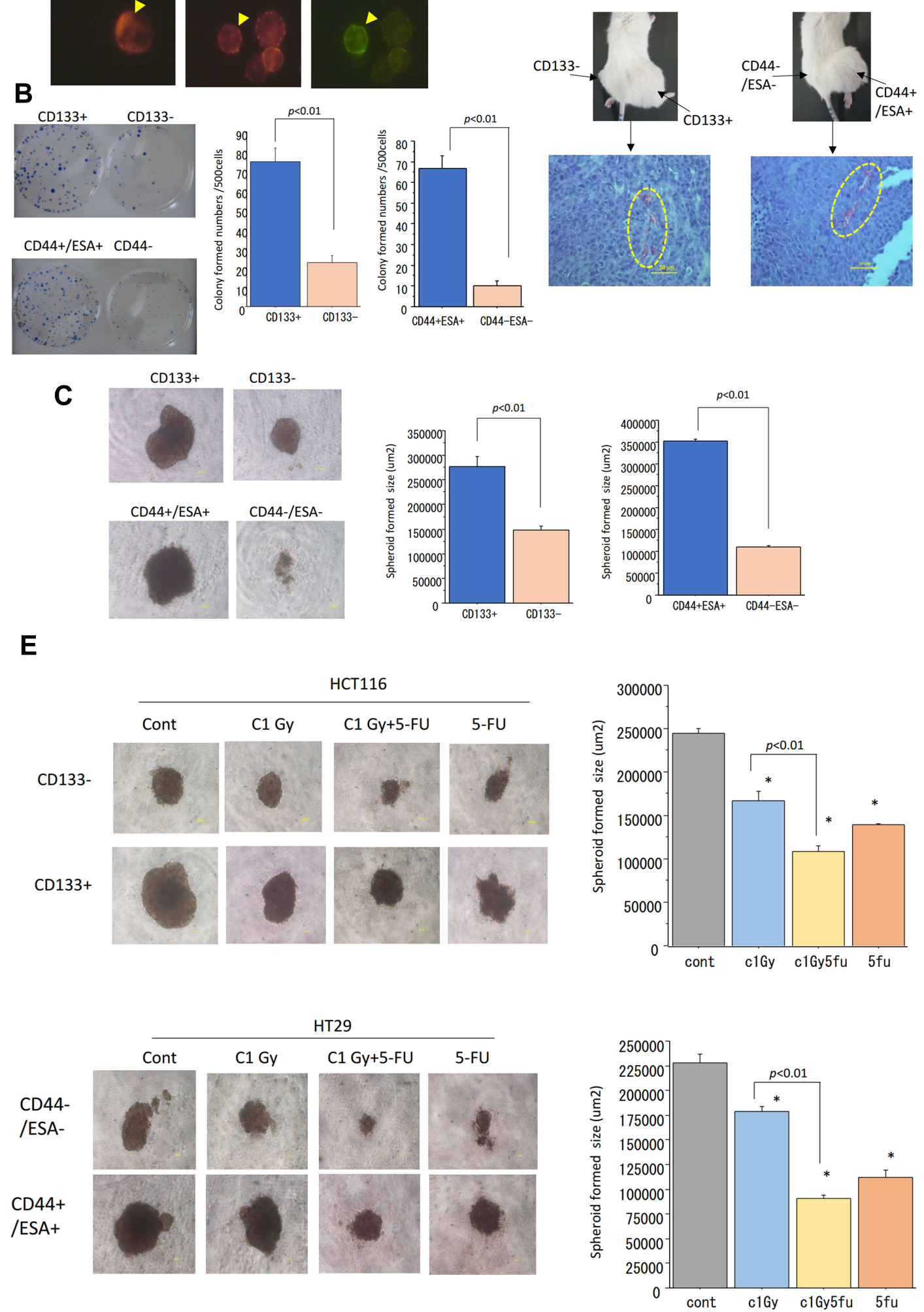

Figure 3 (A) Representative immunofluorescence staining of CDI33+, CD44+, and ESA+ cells in HCTI I6. (B) Colony formation of CDI33+, CD44+/ESA+ and CDI33-, CD44-/ESA- cells isolated from HCTI I6. (C) Spheroid formation of CDI33+, CD44+/ESA+ and CDI33-, CD44-/ESA- cells isolated from HCTII6. (D) Tumorigenicity of CDI33+, CD44+/ESA+ and CDI33-, CD44-/ESA- cells isolated from HCTII6. Histopathological features of xenograft tumor were showed. Dotted circle indicates tumor vessels (red blood cells were seen). (E) Spheroid forming ability after carbon-ion beam irradiation alone, or in combination with 5-FU. *, p<0.0I compared to control. 
with 5-FU. As shown in Figure 4A, carbon-ion beam irradiation significantly increased the expression of the apoptosis-related genes, $\mathrm{Bax}$ and $\mathrm{Bcl} 2$, compared to $\mathrm{X}$-ray irradiation, and the expression was further increased when the carbon-ion beam was combined with 5-FU in HCT116 cells. Expression of autophagy-related genes, including Beclin1 and ATG7, but not LC3, was significantly enhanced following carbon-ion beam irradiation compared to X-ray irradiation, and further augmented when the carbon-ion beam was combination with 5-FU (Figure 4A). The changes in the expression of multiple genes in CSCs and non-CSCs were also examined when treated with carbon-ion beam irradiation alone or in combination with 5-FU. Interestingly, the expression of apoptosis-related gene, Bax was significantly increased after treatment with carbon-ion beam irradiation alone, and further increased when carbon-ion beam irradiation was combined with 5-FU in non-CSCs, but not in CSCs (Figure 4B). In comparison, Bcl2 expression was significantly increased after treatment with carbon-ion beam irradiation alone, and further increased when carbon-ion beam irradiation was combined with 5-FU in CSCs, but not in non-CSCs. Expression of autophagy-related genes, Beclin1, LC3, and ATG7, was significantly enhanced after treatment with combination of carbon-ion beam irradiation and 5-FU in both non-CSCs and CSCs, and especially expression of Beclin1 and ATG7 was enormously increased in non-CSCs (Figure 4B).

\section{Histopathological Changes in Xenograft Tumor Following Carbon-Ion Beam Alone or in Combination with 5-FU}

To investigate the histopathological changes in HCT116 xenograft tumors in vivo following carbon-ion beam irradiation alone or in combination with 5-FU, the tissues were stained with hematoxylin and eosin (HE). HE analysis revealed that the tumor cells were markedly affected when irradiation with 25 Gy of carbon-ion beam combined with $50 \mathrm{mg} / \mathrm{kg}$ 5-FU administered i.p accompanied with necrosis, cavitation and fibrosis compared to irradiation with 30 Gy of carbon-ion beam alone or 5-FU treatment alone (Figure 5).

\section{Discussion}

First, to clarify the cytotoxicity of 5-FU in CRC cells, $\mathrm{CRC}$ cells were treated with increasing concentrations of 5 -FU for various time points. We found that the viability of both HCT116 and HT29 cells was significantly decreased 48 and $72 \mathrm{~h}$ after treatment with 5-FU in a dosedependent manner. To examine whether the cell killing mediated by 5-FU affects carbon-ion beam, CRC cells were treated with carbon-ion beam irradiation alone or in combination with 5-FU. As expected, carbon-ion beam irradiation significantly reduced $\mathrm{CRC}$ cell viability, which was reduced even further when treated in combination with 5-FU. These results are consistent with previous reports showing that 5-FU has radiosensitizing effects in many cancer types such as colorectal, pancreatic and head and neck cancers. ${ }^{37-39}$

It has been demonstrated that 5-FU radiosensitizes CRC cells accompanied with apoptosis and/or autophagy. ${ }^{37,38}$ In this study, we found that carbon-ion beam irradiation significantly enhanced apoptosis in a dose-dependent manner, and induction of both early and total apoptosis markedly increased when the irradiation was combined with 5-FU in HCT116 and HT29 cells. This finding is partially in line with previous reports showing that 5-FU induces apoptosis in gastric, breast and colon cancer cells. ${ }^{40-42}$ To investigate molecular mechanisms of cell death induced by carbon-ion beam alone or in combination with 5-FU, we analyzed changes in the expression of apoptosis- and autophagy-related genes using qPCR. We found that carbon-ion beam irradiation caused significant increase in the apoptosis-related genes, Bax and $\mathrm{Bcl} 2$ compared to X-ray irradiation which was increased even further when the carbon-ion beam was combined with 5-FU in HCT116 cells. Expression of autophagy-related genes, including Beclin1 and ATG7, but not LC3, was significantly enhanced following carbonion beam irradiation compared to X-ray irradiation and was further enhanced when the beam was combined with 5-FU. Our findings are in agreement with previous reports that 5-FU induces not only apoptosis but also autophagy and that both these effects facilitate the anticancer effects, ${ }^{43-45}$ and consequently sensitize CRC cells to carbon-ion beam irradiation.

Using clonogenic assay, we previously found that CSCs derived from CRC cells are resistant to both X-rays and carbon-ion beams compared to non-CSCs. ${ }^{30}$ In this study, the spheroid forming capacity was used to investigate the effect of carbon-ion beams in combination with 5-FU on CSCs. Interestingly, the spheroid forming ability of CSCs was markedly reduced when treated with carbon-ion beam combined with 5-FU compared to that of carbon-ion beam irradiation alone or X-ray irradiation combined with 5-FU. 


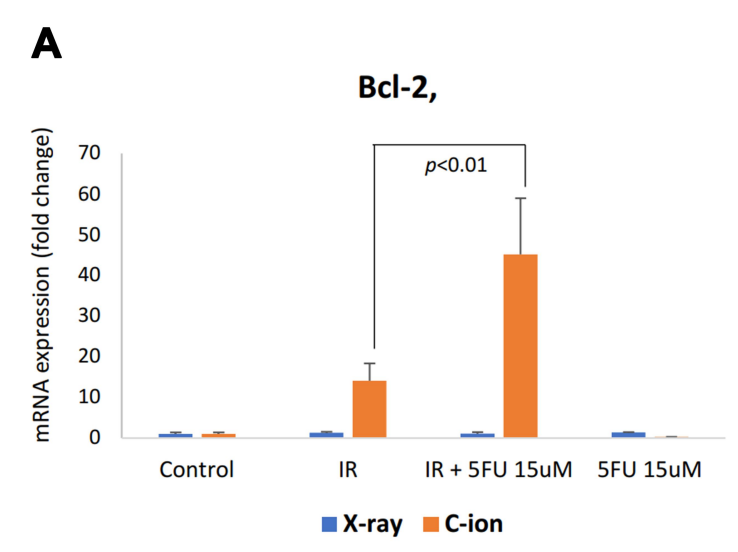

HCT116
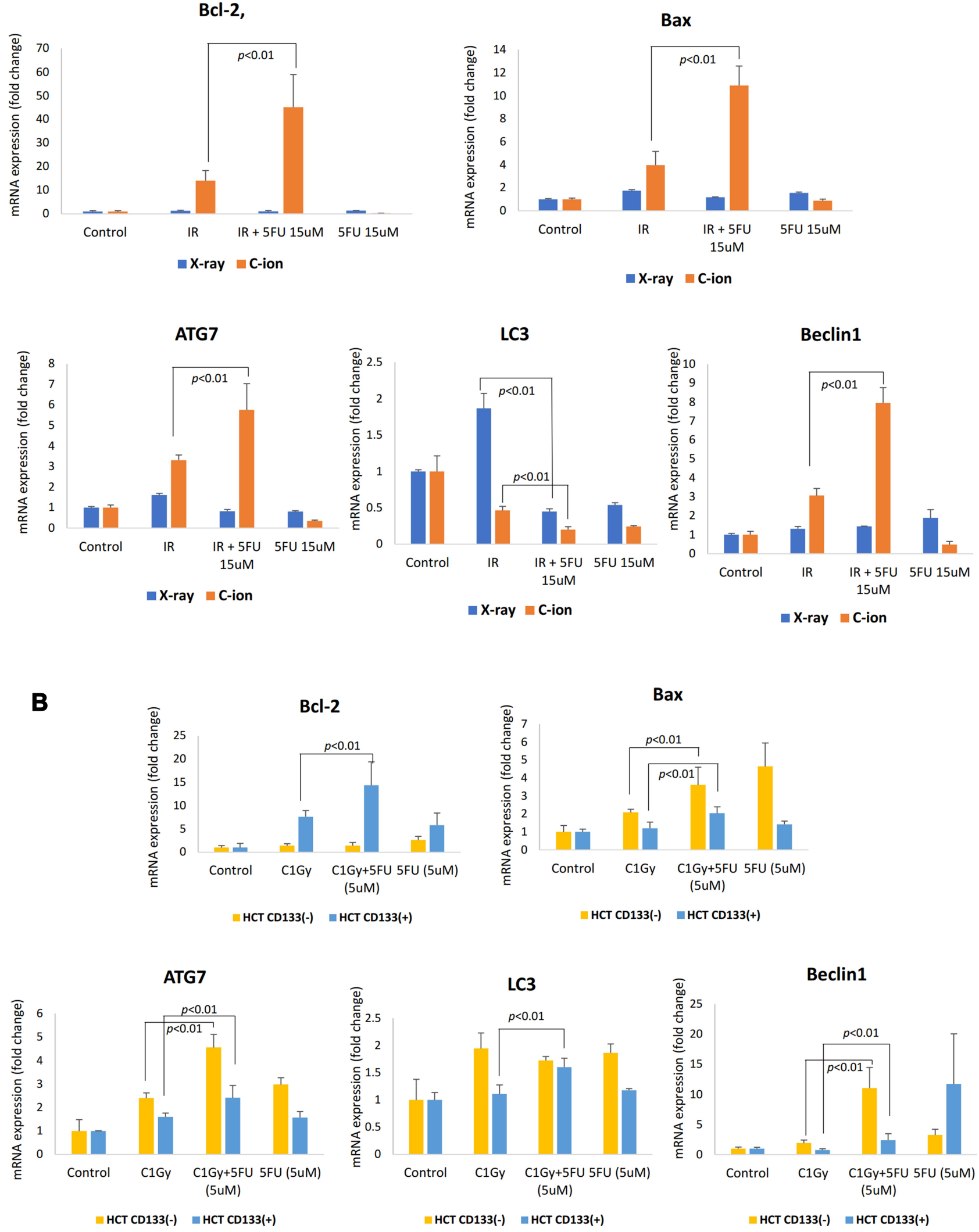

Figure 4 (A) Changes in the expression of apoptosis- and autophagy-related genes $72 \mathrm{~h}$ after carbon-ion beam irradiation alone or in combination with 5 -FU in unsorted HCTII 6 cells. (B) Changes in the expression of apoptosis- and autophagy-related genes $5 \mathrm{~d}$ after carbon-ion beam irradiation (I Gy) alone or in combination with 5-FU $(5 \mu \mathrm{M})$ in CSCs and non-CSCs isolated from HCTII6. 

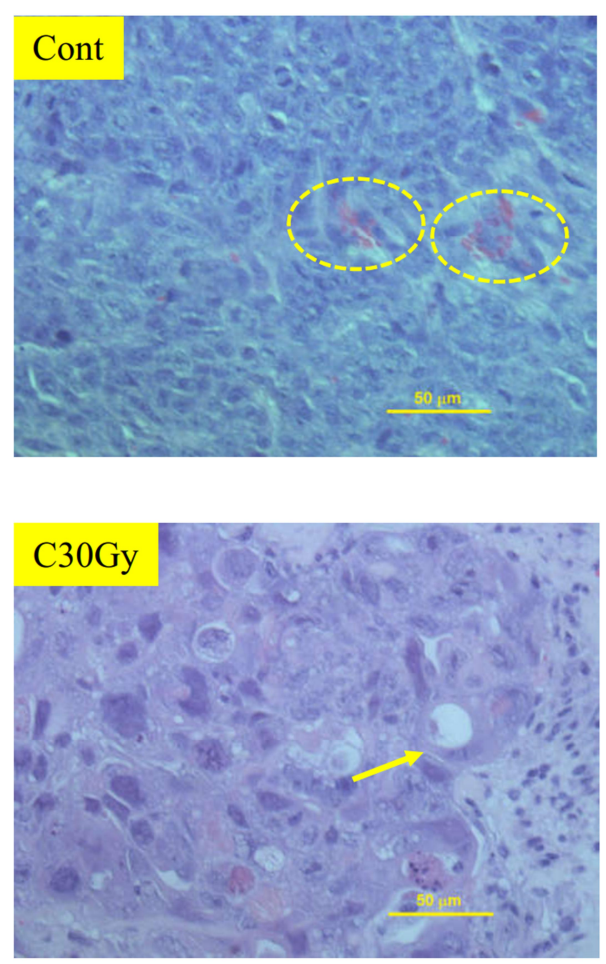
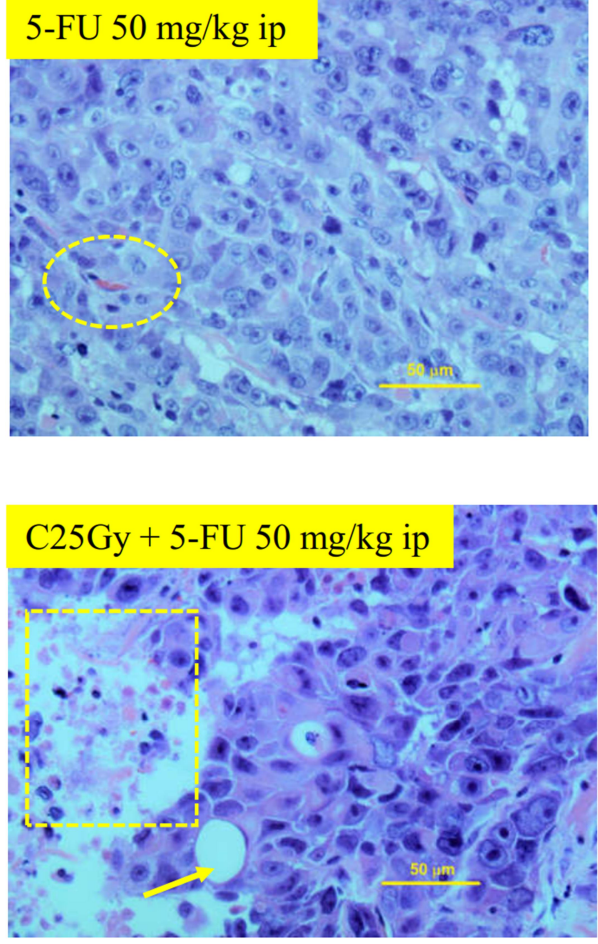

Magnification x 400

Figure 5 Histopathological changes in HCTII 6 xenograft tumor after carbon-ion beam irradiation alone or in combination with 5-FU. Dotted circles indicate tumor vessels (red blood cells were seen), dotted square indicates necrosis, arrows indicate cavitation.

This is partially in line with our previous reports that carbonion beam combined with DNA damaging drugs can effectively destroy CSCs in pancreatic cancer, breast cancer and malignant mesothelioma. ${ }^{32-34}$ We also analyzed the changes in expression of apoptosis- and autophagy-related genes in CSC derived spheroids and non-CSC derived spheroids following treatment with carbon-ion beam irradiation alone or in combination with 5-FU. We found that carbon-ion beam irradiation alone or in combination with 5-FU markedly increased the expression of the apoptosis-related gene, Bax, in non-CSC derived spheroids, whereas the expression of anti-apoptosis-related gene, $\mathrm{Bcl} 2$, was highly enhanced in the CSC derived spheroids, suggesting that carbon-ion beam irradiation alone induced apoptosis in both non-CSCs and CSCs, and it was more significant when carbon-ion beam irradiation combined with 5-FU. Interestingly, the expression of the autophagy-related genes, ATG7, LC3 and Beclin1was tremendously enhanced in non-CSCs following treatment with carbon-ion beam irradiation alone or in combination with 5-FU. Only the combination treatment of carbon-ion beam irradiation and 5-FU significantly increased the expression of all three genes, AGT7, LC3 and Beclin1 in CSCs, whereas carbon-ion beam irradiation alone did not. These findings imply that carbon-ion beam irradiation combined with 5-FU has strong potential to induce autophagy-mediated cell death rather than apoptosis-mediated cell death. This is partially consistent with previous reports that autophagic cell death induced by 5-FU can be used as an alternative cell death pathway in apoptosis defective cells. ${ }^{45}$ In addition, in vivo histopathological analysis of xenograft tumor showed that carbon-ion beam in combination with 5-FU killed tumor cells as evidenced by increased necrosis, cavitation and fibrosis compared to carbon-ion beam irradiation or 5-FU alone.

In summary, carbon-ion beam irradiation combined with 5-FU has superior effects on targeting colorectal CSCs accompanied with increased apoptosis, autophagy, and subsequent cell death compared to carbon-ion beam alone. Taken together, our findings imply that carbon-ion beam combined with 5 -FU has strong potential to kill radioresistant $\mathrm{CRC}$ cells including $\mathrm{CSCs}$ in vitro and in vivo.

\section{Acknowledgments}

We are grateful to Mr. Yamada $\mathrm{T}$ for the kind technical support. 


\section{Disclosure}

The authors report no conflicts of interest for this work.

\section{References}

1. Ryuk JP, Choi GS, Park JS, et al. Ann Surg Treat Res. 2014;86 (3):143-151. doi:10.4174/astr.2014.86.3.143

2. Hashiguchi Y, Muro K, Saito Y, et al. Japanese Society for Cancer of the Colon and Rectum (JSCCR) guidelines 2019 for the treatment of colorectal cancer. Int J Clin Oncol. 2020;25(1):1-42.

3. Westberg K, Palmer G, Hjern F, Johansson H, Holm T, Martling A. Management and prognosis of locally recurrent rectal cancer a national population-based study. Eur J Surg Oncol. 2018;44 (1):100-107. doi:10.1016/j.ejso.2017.11.013

4. Guyot F, Faivre J, Manfredi S, Meny B, Bonithon-Kopp C, Bouvier AM. Time trends in the treatment and survival of recurrences from colorectal cancer. Ann Oncol. 2005;16(5):756-761. doi:10.1093/annonc/mdi151

5. Wong CS, Cummings BJ, Keane TJ, Dobrowsky W, O'Sullivan B, Catton CN. Combined radiation therapy, mitomycin $\mathrm{C}$, and 5-fluorouracil for locally recurrent rectal carcinoma: results of a pilot study. Int J Radiat Oncol Biol Phys. 1991;21(5):1291-1296. doi:10.1016/0360-3016(91)90288-F

6. Farhat W, Azzaza M, Mizouni A, et al. Factors predicting recurrence after curative resection for rectal cancer: a 16-year study. World J Surg Oncol. 2019;17(1):173. doi:10.1186/s12957-019-1718-1

7. Short SS, Stojadinovic A, Nissan A, et al. Adjuvant treatment of early colon cancer with micrometastases: results of a national survey. J Surg Oncol. 2012;106(2):119-122. doi:10.1002/jso.23057

8. Lips DJ, Koebrugge B, Liefers GJ, et al. The influence of micrometastases on prognosis and survival in stage I-II colon cancer patients: the Enroute $\oplus$ Study. BMC Surg. 2011;11:11. doi:10.1186/ 1471-2482-11-11.

9. Harris CA, Solomon MJ, Heriot AG, et al. Patterns of treatment failure after surgery for locally recurrent rectal cancer. Ann Surg. 2016;264(2):323-329. doi:10.1097/SLA.0000000000001524

10. Renehan AG. Techniques and outcome of surgery for locally advanced and local recurrent rectal cancer. Clin Oncol ( $R$ Coll Radiol). 2016;28(2):103-115. doi:10.1016/j.clon.2015.11.006

11. Grothey A, Sobrero AF, Shields AF, et al. Duration of adjuvant chemotherapy for Stage III colon cancer. $N$ Engl J Med. 2018;378 (13):1177-1188. doi:10.1056/NEJMoa1713709

12. Neugut AI, Matasar M, Wang X, et al. Duration of adjuvant chemotherapy for colon cancer and survival among the elderly. $J$ Clin Oncol. 2006;24(15):2368-2375. doi:10.1200/JCO.2005.04.5005

13. Kornmann M, Formentini A, Ette C, et al. Prognostic factors influencing the survival of patients with colon cancer receiving adjuvant 5-FU treatment. Eur J Surg Oncol. 2008;34(12):1316-1321. doi:10.1016/j.ejso.2008.01.019

14. Loree JM, Cheung WY. Optimizing adjuvant therapy and survivorship care of stage III colon cancer. Future Oncol. 2016;12 (17):2021-2035. doi:10.2217/fon-2016-0109

15. Gustavsson B, Carlsson G, Machover D, et al. A review of the evolution of systemic chemotherapy in the management of colorectal cancer. Clin Colorectal Cancer. 2015;14(1):1-10. doi:10.1016/j. clcc.2014.11.002

16. O'Brien CA, Pollett A, Gallinger S, et al. A human colon cancer cell capable of initiating tumour growth in immunodeficient mice. Nature. 2007;445:106-110. doi:10.1038/nature05372

17. Ricci-Vitiani L, Lombardi DG, Pilozzi E, et al. Identification and expansion of human colon-cancer-initiating cells. Nature. 2007;445 (7123):111-115. doi:10.1038/nature05384

18. Bao S, Wu Q, McLendon RE, et al. Glioma stem cells promote radioresistance by preferential activation of the DNA damage response. Nature. 2006;444(7120):756-760. doi:10.1038/nature05236
19. Hambardzumyan D, Squatrito M, Holland EC. Radiation resistance and stem-like cells in brain tumors. Cancer Cell. 2006;10:454-456. doi:10.1016/j.ccr.2006.11.008

20. Rich JN. Cancer stem cells in radiation resistance. Cancer Res. 2007;67:8980-8984. doi:10.1158/0008-5472.CAN-07-0895

21. Elbadawy M, Usui T, Yamawaki H, Sasaki K. Emerging roles of c-myc in cancer stem cell-related signaling and resistance to cancer chemotherapy: a potential therapeutic target against colorectal cancer. Int J Mol Sci. 2019;20(9):2340. doi:10.3390/ijms20092340

22. Abugomaa A, Elbadawy M, Yamawaki H, Usui T, Sasaki K. Emerging roles of cancer stem cells in bladder cancer progression, tumorigenesis, and resistance to chemotherapy: a potential therapeutic target for bladder cancer. Cells. 2020;9(1):23. doi:10.3390/cells 9010235

23. Abugomaa A, Elbadawy M. Patient-derived organoid analysis of drug resistance in precision medicine: is there a value? Expert Rev Precis Med Drug Dev. 2020;5(1):1-5. doi:10.1080/23808993.2020.1715794

24. Baumann M, Krause M, Hill R. Exploring the role of cancer stem cells in radioresistance. Nat Rev Cancer. 2008;8:545-554. doi:10.1038/nrc2419

25. Diehn M, Clarke MF. Cancer stem cells and radiotherapy: new insights into tumor radioresistance. J Natl Cancer Inst. 2006;98 (24):1755-1757. doi:10.1093/jnci/djj505

26. Dingli D, Michor F. Successful therapy must eradicate cancer stem cells. Stem Cells. 2006;24(12):2603-2610. doi:10.1634/stemcells.200 6-0136

27. Shinoto M, Yamada S, Okamoto M, et al. Carbon-ion radiotherapy for locally recurrent rectal cancer: japan Carbon-ion Radiation Oncology Study Group (J-CROS) study 1404 rectum. Radiother Oncol. 2019;132:236-240. doi:10.1016/j.radonc.2018.10.007

28. Yamada S, Kamada T, Ebner DK, et al. Working group on locally recurrent rectal cancer. carbon-ion radiation therapy for pelvic recurrence of rectal cancer. Int J Radiat Oncol Biol Phys. 2016;96 (1):93-101. doi:10.1016/j.ijrobp.2016.04.022

29. Shiba S, Okamoto M, Kiyohara H, et al. Prospective observational study of high-dose carbon-ion radiotherapy for pelvic recurrence of rectal cancer (GUNMA 0801). Front Oncol. 2019;9:702. doi:10.33 89/fonc. 2019.00702.

30. Cui X, Oonishi K, Tsujii H, et al. Effects of carbon ion beam on putative colon cancer stem cells and its comparison with X-rays. Cancer Res. 2011;71:3676-3687. doi:10.1158/0008-5472.CAN-10-2926

31. Oonishi K, Cui X, Hirakawa H, et al. Different effects of carbon ion beams and X-rays on clonogenic survival and DNA repair in human pancreatic cancer stem-like cells. Radiother Oncol. 2012;105 (2):258-265. doi:10.1016/j.radonc.2012.08.009

32. Sai S, Wakai T, Vares G, et al. Combination of carbon ion beam and gemcitabine causes unreparable DNA damage and death of radioresistant pancreatic cancer stem-like cells in vitro and in vivo. Oncotarget. 2015;6:5517-5535. doi:10.18632/oncotarget.3584

33. Sai S, Vares G, Kim EH, et al. Carbon ion beam combined with cisplatin effectively disrupts triple negative breast cancer stem-like cells in vitro. Mol Cancer. 2015;14:166. doi:10.1186/s12943-015-0429-7.

34. Sai S, Suzuki M, Kim EH, Hayahsi M, Yamamoto N, Miyamoto T. Effects of carbon ion beam alone or in combination with cisplatin on malignant mesothelioma cells in vitro. Oncotarget. 2018;9(19): 14849-14861. doi:10.18632/oncotarget.23756

35. Sai S, Kim EH, Suzuki M, Horimoto Y, Hayashi M. Combination of carbon-ion beam and dual tyrosine kinase inhibitor, lapatinib, effectively destroys HER 2 positive breast cancer stem-like cells. Am J Cancer Res. 2020;10(8):2371-2386.

36. Kim EH, Kim MS, Takahashi A, et al. Carbon-ion beam irradiation alone or in combination with zoledronic acid effectively kills osteosarcoma cells. Cancers. 2020;12:698.

37. Smalley SR, Kimler BF, Evans RG. 5-Fluorouracil modulation of radiosensitivity in cultured human carcinoma cells. Int $J$ Radiat Oncol Biol Phys. 1991;20(2):207-211. doi:10.1016/0360-3016(91) 90091-H 
38. Urick ME, Chung EJ, Shield WP 3rd, et al. Enhancement of 5-fluorouracil-induced in vitro and in vivo radiosensitization with MEK inhibition. Clin Cancer Res. 2011;17(15):5038-5047. doi:10.11 58/1078-0432.CCR-11-0358

39. Balart J, Capellà G, de Los Inocentes RM, et al. Treatment with 5-fluorouracil enhances radiosensitivity of the human pancreatic cancer cell line MiaPaCa-2. Pancreatology. 2002;2(1):40-45. doi:10.1159/000049447

40. Fu KK. Radiation therapy with 5-fluorouracil in head and neck cancer. Semin Radiat Oncol. 1997;7(4):274-282.

41. Nita ME, Nagawa H, Tominaga O, et al. 5-Fluorouracil induces apoptosis in human colon cancer cell lines with modulation of Bcl-2 family proteins. Br J Cancer. 1998;78(8):986-992. doi:10.1038/bjc.1998.617

42. Osaki M, Tatebe S, Goto A, Hayashi H, Oshimura M, Ito H. 5-Fluorouracil (5-FU) induced apoptosis in gastric cancer cell lines: role of the p53 gene. Apoptosis. 1997;2(2):221-226. doi:10.1023/A: 1026476801463
43. Tang JC, Feng YL, Liang X, Cai XJ. Autophagy in 5-fluorouracil therapy in gastrointestinal cancer: trends and challenges. Chin Med J (Engl). 2016;129(4):456-463. doi:10.4103/03666999.176069

44. Yang JW, Zhang QH, Liu T. Autophagy facilitates anticancer effect of 5-fluorouracil in HCT-116 cells. J Cancer Res Ther. 2018;14(Supplement):S1141-S1147. doi:10.4103/0973-1482.2 04898

45. Xiong HY, Guo XL, Bu XX, et al. Autophagic cell death induced by 5-FU in Bax or PUMA deficient human colon cancer cell. Cancer Lett. 2010;288(1):68-74. doi:10.1016/j.canlet.2009.06.039

\section{Publish your work in this journal}

OncoTargets and Therapy is an international, peer-reviewed, open access journal focusing on the pathological basis of all cancers, potential targets for therapy and treatment protocols employed to improve the management of cancer patients. The journal also focuses on the impact of management programs and new therapeutic agents and protocols on patient perspectives such as quality of life, adherence and satisfaction. The manuscript management system is completely online and includes a very quick and fair peer-review system, which is all easy to use. Visit http://www.dovepress.com/ testimonials.php to read real quotes from published authors. 